\title{
Study of Serum Magnesium Level in Acute Diarrhea
}

\author{
NIHARPATEL ${ }^{1 \cdot}$ PRADIP VEKARIYA ${ }^{2}$,Rakesh Bharodiya ${ }^{3}$,Dr.Vipul \\ Srivastav $^{4}$ \\ ${ }^{1,2}$ Resident doctor, Department of Internal Medicine, SMIMER, Surat \\ ${ }^{3}$ Assistant Professor, Department of Internal Medicine, SMIMER, Surat \\ ${ }^{4}$ Professor and Head, Department of Internal Medicine, SMIMER, Surat
}

\author{
Abstract: \\ The purpose of this study To study serum magnesium levels in acute gastroenteritis. \\ 2. Correlation of severity of diarrhea with serum magnesium level. \\ 3. Correlation of serum magnesium level in diarrhea with serumpotassium and calcium levels. \\ 4. To correlate the severity of hypomagnesemia if present with itsclinical and ECG manifestationsTo study \\ serum magnesium levels in acute gastroenteritis. \\ 2. Correlation of severity of diarrhea with serum magnesium level. \\ 3. Correlation of serum magnesium level in diarrhea with serumpotassium and calcium levels. \\ 4. To correlate the severity of hypomagnesemia if present with its \\ clinical and ECG manif
}

\section{Background}

Magnesium is an essential element in biological systems \& is present in every cell type in every organism. It is the $4^{\text {th }}$ most common cation in the body after sodium, potassium \& calcium. Also, the second most common divalent cation in the body after calcium. Adenosine triphosphate, the main source of energy in cells, must be bound to a magnesium ion in order to be biologically active. What is called adenosine triphosphate (ATP) is often actually magnesium-adenosine triphosphate (Mg-ATP).Normal concentration of extracellular magnesium and calcium are crucial for normal neuromuscular activity. Magnesium is required for normal functioning of $\mathrm{Na}+\mathrm{K}+\mathrm{ATPase}$ activity, which is necessary for maintenance of normal potassium level. It also has a role in production of cAMP, by which it regulates the release \& function of parathyroid hormone. Deficiency of $\mathrm{Mg}$ ultimately leads to hypocalcemia.Over 300 enzymes require the presence of magnesium ions for their catalytic action, including all enzymes utilizing or synthesizing ATP, or those that use other nucleotides to synthesize DNA and RNA.Half of total body $\mathrm{Mg}$ is located in bone. Almost all extraskeletal magnesium is present within cells, only $1 \%$ of body magnesium resides in the extra-cellular fluid. Only $5-10 \%$ of intracellular magnesium is free, which is essential for regulation of intracellular magnesium content. The concentration of magnesium in serum is closely regulated by the intestine and kidney within the range of 1.6 to $2.3 \mathrm{mg} / \mathrm{dl}(0.7-1.1 \mathrm{mmol} / \mathrm{L})$. Hypomagnesemia can result from intestinal malabsorption, protracted vomiting, diarrhea, or intestinal drainage, defective renal tubular magnesium reabsorption, or rapid shift of magnesium from ECF into cells, bones or third space. ${ }^{3,4}$ Hypomagnesemia may cause generalized alteration in neuromuscular function including tetany, tremors, seizure, muscle weakness, ataxia, nystagmus, vertigo, apathy, depression, irritability, delirium and psychosis. Sinus tachycardia as well as cardiac arrhythmias like supra ventricular tachycardias and ventricular arrhythmias may occur. ECG abnormalities may include prolonged PR or QT interval, T wave flattening or inversion and ST straightening. Sensitivity to digitalis toxicity may be enhanced. All these can result in sudden death. ${ }^{5}$

Other electrolyte abnormalities often coinciding with hypomagnesemia include hypocalcemia and hypokalemia which may not be easily corrected unless magnesium is corrected as well. ${ }^{6}$

Diarrhea is loosely defined as passage of abnormally liquid or unformed stools at an increased frequency. Stool weight $>200 \mathrm{gm} /$ day can generally be considered as diarrheal ${ }^{1}$. Acute diarrhea is defined as diarrhea that lasts less than 2 weeks. As the bowel is stimulated by the organism and its toxin, the intestinal tract secretes water and electrolytes in the intestinal lumen. All electrolytes are thus variably lost in the diarrheal fluid, mainly $\mathrm{Na}^{+}, \mathrm{K}^{+}, \mathrm{Cl}^{-}, \mathrm{HCO}^{-}$. Also, sodium and water reabsorption in the bowel is inhibited. Due to loss of sodium and water in diarrhea, body tries to retain sodium under the influence of aldosterone. Aldosterone acts on collecting tubules in kidney which upregulates the sodium channels \& ultimately leads to increased sodium absorption. To maintain intracellular $\mathrm{pH}$, other cation like $\mathrm{K}^{+}$and $\mathrm{Mg}^{+}$are pushed out of the cells \& lost in urine. This aggravates the hypokalemia \& hypomagnesemia.

Several studies have focused on the etiology and electrolyte disturbances in hospitalized patients with acute diarrhea. However, studies regarding serum magnesium level among the hospitalizations associated with 
gastroenteritis in adults remain least explored and not well investigated so far even though it imposes a major effect on other electrolyte levels leading to neurological and cardiovascular manifestations.

Considering the above facts, the present study was undertaken to study the serum magnesium level and its correlation with other electrolyte disturbances among adult patients with acute gastroenteritis. The study will also provide data for recommendations concerning routine testing panel to guide clinicians in the management of patient of acute diarrhea and gastroenteritis.

Study Type

\section{Materials And Methods}

This is a case control observational study.

\section{Study Settings}

This study was conducted in the city of Surat at department of general medicine in the Surat Municipal Institute of Medical Education and Research in the year 2012-13. Cases included adult patients admitted with acute diarrhea while controls were healthy individuals who voluntarily participated in the study.

\section{Ethical Considerations}

Permission was obtained from the Institutional Ethical Committee of the Surat Municipal Institute of Medical Education and Research, Surat before commencing of the study. Confidentiality of the data collected was maintained strictly throughout the study.

\section{Inclusion Criteria For Patients}

- Patient with Acute diarrhea defined as 3 or more loose, watery stools in a day, of less than 2 weeks duration)

- Age above 18 years

\section{Exclusion criteria for patients:}

- Patients already receiving any form of calcium or magnesium therapy

- Liver disease

- Hypoalbuminemia due to any cause

- Patient with pre existing renal failure, Co-morbid conditions like malignancy, diabetes mellitus, hypertension, malnutrition and wasting, diarrhea attributable to any other systemic illness.

\section{Inclusion Criteria For Control}

- Healthy individuals above 18 years age

\section{Exclusion Criteria For Control}

- Patients already receiving any form of calcium or magnesium therapy

- Liver disease

- Hypoalbuminemia due to any cause

- Patient with pre existing renal failure, Co-morbid conditions like malignancy, diabetes mellitus, hypertension, malnutrition and wasting, diarrhea attributable to any other systemic illness.

- Patient with any other acute illness.

According to the above inclusion criteria, total 100 patients of Acute Diarrhea \& 50 controls were enrolled in to the study. Detailed clinical evaluation, as per the annexed proforma [page no.82] was done in all the enrolled patients. Laboratory investigations were done on admission and repeated periodically as advised by the consulting physician. All investigations were done in the pathology, biochemistry \& microbiology labs of SMIMER.

\section{Results}

The study was carried out at a tertiary care centre at SMIMER, surat over a duration of one year in which serum magnesium and other electrolyte of cases with acute diarrhea \& healthy controls were studied and analysed. Total of 100 cases \& 100 controls were studied. There were 71 males and 29 females in cases of acute diarrhea. Males affected more as compared to females.Maximum numbers of cases were between the ages of $21-50$ years. The mean age was $35.67 .68 \%$ cases of acute diarrhea had signs of moderate dehydration, $10 \%$ cases had signs of severe dehydration with $22 \%$ had mild dehydration on admission. $55 \%$ cases of acute 
diarrhea had hypomagnasemia on admission and 6\% cases of acute diarrhea had severe hypomagnasemia. 39\% cases of acute diarrhea had hypokalemia on admission and $22 \%$ cases had severe hypokalemia.

12 (12\%) cases had abnormal stool examination report. Repots of $9(9 \%)$ cases had no organism in stool but had presence of pus cells, suggestive of bacterial infection. $2(2 \%)$ had presence of mucous which was suggestive of dysentery. 1 (1\%) had presence of ova \& cyst of hookworms, suggestive of parasitic infection. In others, 88 $(88 \%)$ cases had no any significant abnormality in stool examination report. Which may suggestive of either viral diarrhea or any other non infective etiology. $24 \%$ cases of acute diarrhea were HIV positive.

Only $10 \%$ cases had symptoms attributable to hypomagnesemia mostly neuromuscular. Hypomagnesemia was found to have a significant association with grades of dehydration. In cases, $60 \%$ of moderate dehydration had low Mg levels, with $4.4 \%$ having severe hypomagnesemia. In cases of acute diarrhea with severe dehydration $80 \%$ had low Mg level with $30 \%$ having severe hypomagnesemia.

Hypomagnesemia had a significant association with hypokalemia in cases of acute diarrhea. With $80 \%$ cases having normal $\mathrm{Mg}$ level had normal potassium levels while 31\% cases of mild hypomagnesemia had hypokalemia, $58.3 \%$ cases of moderate hypomagnesemia had hypokalemia, $83 \%$ cases of severe hypomagnesemia had hypokalemia with $66.7 \%$ cases in this group had severe hypokalemia. Calcium levels did not have significant association with grades of dehydration in cases of acute diarrhea. Low calcium levels were found in cases, with hypomagnesemia with $\mathrm{p}$ of 0.03 . The association was weak in cases in our study as compared to reference study by Samiullah et al where the association was $\mathrm{p}<0.001$, there the cut off value of calcium $<9.0 \mathrm{mg} / \mathrm{dl}$ was taken for hypocalcemia.

Grade of dehydration had significant correlation with potassium levels with coefficient of -0.307 . Grade of dehydration also had a significant correlation with $\mathrm{Mg}$ levels with coefficient of $-0.356 . \mathrm{K}+$ also had significant correlation with $\mathrm{Mg}$ levels with coefficient of 0.466. 6 (6\%) cases of acute diarrhea had abnormal ECG findings. $66.7 \%$ (4) cases of severe hypomagnesemia had abnormal ECG. There is significant association between hypomagnesemia and ECG changes, but as the severe hypomagnesemia is also associated with other electrolyte disturbance, we can't say that ECG changes are due to hypomagnesemia only.

\section{Discussion}

The present study was conducted in a tertiary care hospital over a period of one year with the aim of studying serum magnesium levels in patients admitted with acute diarrhea and healthy controls, correlating the severity of dehydration with serum magnesium levels, correlation of serum magnesium levels with serum potassium and calcium levels and finally correlating severity of hypomagnesemia with the clinical manifestations of hypomagnesemia. Cases were subdivided by grades of dehydration by their clinical manifestations into mild, moderate and severe dehydration. Total of 100 cases of acute diarrhea \& 100 controls were studied.

One hundred cases of acute diarrhea were selected as per the inclusion criteria mentioned in the materials and methods. The observations are compared with the studies done by others on the same subject.

Age and sex distribution

\begin{tabular}{|c|c|c|c|c|c|c|c|c|c|c|c|c|c|c|c|c|c|}
\hline \multicolumn{5}{|c|}{ A g e i n y e a r s } & \multicolumn{6}{|c|}{ Pres e n t s t ud y } & \multicolumn{7}{|c|}{ S a m i u l l a h e $t$ a l } \\
\hline & & & & & \multicolumn{3}{|c|}{ C a s e $\mathrm{s}$} & \multicolumn{3}{|c|}{ Percentage } & & 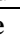 & $\mathbf{S}$ & \multicolumn{4}{|c|}{ Percentage } \\
\hline$<$ & & 2 & & 0 & 1 & & 0 & 1 & 0 & $\%$ & \multicolumn{3}{|c|}{8} & 1 & & 2 & $\%$ \\
\hline 2 & 1 & - & 3 & $\mathbf{0}$ & 3 & & 4 & 3 & 4 & $\%$ & 1 & & 8 & 2 & & 3 & $\%$ \\
\hline 3 & 1 & - & 4 & $\mathbf{0}$ & 2 & & 7 & 2 & 7 & $\%$ & 1 & & 6 & 2 & & $t$ & $\%$ \\
\hline 4 & 1 & - & 5 & 0 & 1 & & 7 & 1 & 7 & $\%$ & 1 & & 6 & 2 & & $t$ & $\%$ \\
\hline$>$ & & 5 & & $\mathbf{0}$ & 1 & & 2 & 1 & 2 & $\%$ & & & & 1 & & 2 & $\%$ \\
\hline $\mathbf{T}$ & o & $\mathbf{t}$ & $\mathbf{a}$ & I & 1 & 0 & 0 & 1 & $\begin{array}{ll}0 & 0\end{array}$ & $\%$ & 6 & & 6 & 1 & 0 & 0 & $\%$ \\
\hline
\end{tabular}

In our study maximum numbers of cases were between the age of 21-50 years, $34(34 \%)$ cases between 21-30 years and $27(27 \%)$ cases between 31-40 years and 17 (17\%) cases between 41-50 years. This correlates well with a study on hypocalcemia in acute gastroenteritis carried out by Bhikha Ram, Samiullah Shaikh, in their study of 66 cases and 66 controls.In our study, out of 100 cases $29(29 \%)$ were females and $71(71 \%)$ were males while in Samiullah et al there were 30 (45\%) females and $36(55 \%)$ males.

Grades of dehydration in acute diarrhea

\begin{tabular}{|c|c|c|c|c|c|c|c|c|c|c|c|c|c|c|}
\hline \multirow[t]{2}{*}{ Grades of deliration } & \multicolumn{6}{|c|}{ Pr e s e n t s t u d y } & \multicolumn{8}{|c|}{ N.K. M i t t a l e t a l } \\
\hline & & $\mathrm{s}$ & $\mathbf{S}$ & & rcent & $\mathrm{ge}$ & & $\mathbf{a}$ & $\mathbf{s}$ & 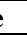 & & & en & ge \\
\hline M & 2 & & 2 & 2 & 2 & $\%$ & 3 & & & & & 6 & 0 & $\%$ \\
\hline Mod e r a t e & 6 & & 8 & 6 & 8 & $\%$ & 1 & & & & & 3 & 0 & $\%$ \\
\hline $\begin{array}{lllll}\mathbf{S} & \mathbf{e} & \mathbf{v} & \mathbf{e} & \mathbf{r} \\
\end{array}$ & 1 & & 0 & 1 & 0 & $\%$ & & & 5 & & & 1 & 0 & $\%$ \\
\hline $\begin{array}{llll}\mathbf{T} & \mathbf{o} & \mathbf{t} & \mathbf{a} \\
\end{array}$ & 1 & 0 & 0 & 1 & $\begin{array}{ll}0 & 0 \\
\end{array}$ & $\%$ & 5 & & & & & 1 & 0 & 0 \\
\hline
\end{tabular}


In our study, all cases showed varying degrees of dehydration. This is probably due to the fact that this study included only inpatients and patients without dehydration or mild diarrhea were not admitted. Among them, mild dehydration was observed in $22(22 \%)$ of cases. Moderate dehydration was observed in $68(68 \%)$ of cases. Severe dehydration was observed in $10(10 \%)$ of cases.

Study by N.K. mittal was carried out in 50 cases of acute diarrhea in pediatric population. ${ }^{55}$ Which shows $60 \%$ cases had mild, $30 \%$ cases had moderate and $10 \%$ cases had severe dehydration. The difference between two groups is probably because of regional influences, different age group or knowledge, attitude and practice of patients.

\section{Magnesium levels}

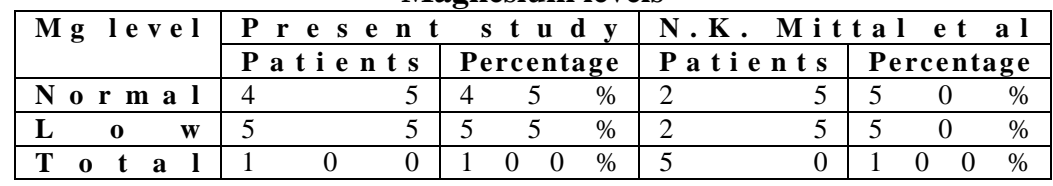

In our study, out of 100 cases, magnesium levels were normal in 45 (45\%) cases. Hypomagnesemia was present in $55(55 \%)$ cases. Among hypomagnesemia, 13\% had mild, 36\% had moderate and $6 \%$ had severe hypomagnesemia.Study on serum and rectal mucosal $\mathrm{Mg}$ status in acute and chronic diarrhea by N.K. Mittal, H.P.S. Sachdev and H.S. yadav had estimated Serum and rectal mucosal magnesium content in children with acute diarrhea (Group I: $n=50$ ), chronic diarrhea (Group II: $n=25$ ), extra-intestinal infections (Group III: $n=$ 15) and healthy controls (Group IV: $n=20$ ). The sex and nutritional status of the different groups were comparable. The mean serum magnesium levels in acute and chronic diarrhoea were comparable to healthy and infected controls. The tissue magnesium content of infants with chronic diarrhoea was significantly $(P<0.001)$ lower than other groups. Repeat estimation at discharge in 38 patients (25 in Group 1, 13 in Group II) revealed a significant reduction in serum levels in both groups $(\mathrm{P}<0.05$ and $\mathrm{P}<0.01$, respectively) and in tissue levels in acute diarrhoea $(\mathrm{f}<0.05)$. So in this study $50 \%$ cases of acute diarrhea had developed hypomagnesemia. ${ }^{55}$

Calcium level

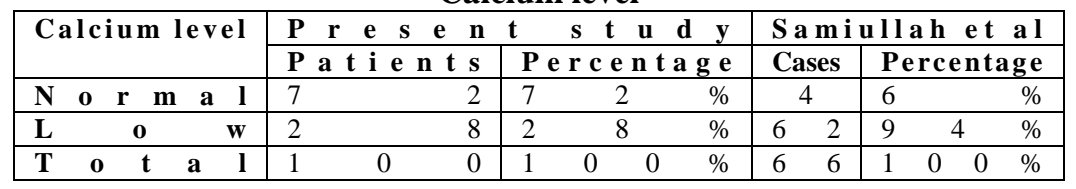

In present study of 100 cases, $28(28 \%)$ had hypocalcemia. While in study, "Hypocalcemia in acute gastroenteritis" by Bhikha Ram, Samiullah Shaikh, in their study of 66 cases and 66 controls, they found hypocalcemia in $94 \%$ cases of acute diarrhea. This difference is because of cut off value for defining hypocalcemia in their study was $<9.0 \mathrm{mg} / \mathrm{dl}$. In our study it was $<8.5 \mathrm{mg} / \mathrm{dl} .{ }^{54} \mathrm{In}$ our study, there was no significant association between calcium level and grades of dehydration in cases of acute diarrhea but hypocalcemia had a significant association with hypomagnesemia (p- value 0.032 ).

\section{Other electrolytes}

\begin{tabular}{|c|c|c|c|c|c|c|c|c|c|c|}
\hline \multirow[t]{2}{*}{ Electrolyte disturbance } & $\mathbf{P}$ & e & $t$ & $s \mathbf{t}$ & d $\mathbf{y}$ & \multicolumn{5}{|c|}{ Shah G.S. e t a l } \\
\hline & \multicolumn{2}{|c|}{ Patients } & \multicolumn{3}{|c|}{ Percentage } & \multicolumn{2}{|c|}{ Cases } & \multicolumn{3}{|c|}{ Percentage } \\
\hline Hy poka le mi a & 3 & 9 & 3 & 9 & $\%$ & 2 & 6 & 4 & 6 & $\%$ \\
\hline Hy ponatremia & 5 & 8 & 5 & 8 & $\%$ & 3 & 2 & 5 & 6 & $\%$ \\
\hline $\begin{array}{l}\text { H y p o k a l e m i a + } \\
\text { Hyponatremia }\end{array}$ & 2 & 2 & 2 & 2 & $\%$ & 1 & 5 & 2 & 6 & $\%$ \\
\hline Hypokalemia+ Hypomagnesemia & 3 & 2 & 3 & 2 & $\%$ & & . & & - & \\
\hline Hypocalcemia+ Hypomagnesemia & 2 & 0 & 2 & 0 & $\%$ & & & & - & \\
\hline
\end{tabular}

In our study, total $39 \%$ cases had hypokalemia, $58 \%$ cases had hyponatremia, $22 \%$ cases had combined hyponatremia with hypokalemia. Among the cases of hypomagnesemia, 32 cases had combined hypokalemia with hypomagnesemia and 20 cases had combined hypocalcemia with hypomagnesemia. Similar results had been seen in study conducted by Shah G.S. et al. this study was conducted in 57 pediatric patients admitted with acute diarrhea. ${ }^{56}$ In our study, serum potassium level had a significant association with grades of dehydration and also had a significant association with serum magnesium level with $\mathrm{p}=0.0003$.In our study, symptoms of hypomagnesemia were mainly neuromuscular including tremors, cramps, myoclonic jerks, muscle spasms (all these patients had normal level of serum calcium).

Inspite of having high incidence of hypomagnesemia in AGE (55\%) only 10 cases (10\%) were having symptoms attributable to hypomagnesemia. From this 10 cases, 1 had mild hypomagnesemia, 2 cases had moderate and 5 had severe hypomagnesemia. 6 cases $(6 \%)$ had muscle cramps(case 7,28,40,50,69,78). Of this 6 
cases, 5 had normal potassium level, only 1 case had hypokalemia which could be contributary to cramps. Hence the cramps could be attributed to hypomagnesemia.

3 cases had myoclonic jerks (case 14,92,93), out of these 2 cases had hypokalemia. Since hypokalemia is not a known cause of myoclonic jerks, also all these 3 cases had normal Ca levels, so the myoclonic jerks could be attributed to hypomagnesemia. 1 case (case 50) had tetany, this patient had $\mathrm{Mg}$ of 1.3 and $\mathrm{Ca}$ of $8.2 \mathrm{mg} \%$ (total calcium). Tetany in this patient could be attributed to hypomagnesemia or hypocalcemia.

\section{Cases Of Acute Diarrhea With Symptoms Possible Due To Hypomagnesemia}

\begin{tabular}{|c|c|c|c|c|c|c|c|}
\hline Case number & $\mathrm{S} y \mathrm{mptom} \mathrm{s}$ & $\mathrm{Mg}^{2+}$ & & & & & + \\
\hline 7 & Muscle cramps & 1.4 & 2 & 8 & 8 & & 9 \\
\hline 4 & Myoclonic jerk & 0.4 & 4 & & 8 & t & 9 \\
\hline 2 & Muscle cramps & 1 & 4 & 4 & 8 & & 8 \\
\hline 0 & Muscle cramps & 1.6 & 5 & 6 & 9 & . & 2 \\
\hline 6 & $\begin{array}{llllll}\mathrm{T} & \mathrm{e} & \mathrm{t} & \mathrm{a} & \mathrm{n} & \mathrm{y} \\
\end{array}$ & 1.3 & 3 & 4 & 8 & & 2 \\
\hline 0 & Muscle cramps & 0.6 & 3 & 1 & 9 & & 1 \\
\hline 6 & Muscle cramps & 1.2 & 4 & 7 & 8 & & 8 \\
\hline 8 & Muscle cramps & 1.6 & 3 & 8 & 9 & & \\
\hline 9 & Myoclonic jerk & 1 & 2 & 7 & 8 & & 5 \\
\hline 9 & Myoclonic jerk & 0.5 & 2 & 9 & 8 & & 8 \\
\hline
\end{tabular}

In all above patients symptoms disappeared after correction of serum magnesium thus reinforcing the need to monitor serum levels of $\mathrm{Mg}$ whenever symptoms suggestive of hypomagnesemia are present.

Magnesium levels are commonly monitored in chronic diarrhea in clinical practice but in acute diarrhea this is hardly ever done, the above results highlight the issue of monitoring serum magnesium levels in AGE on routine basis. To apply these facts on entire population large scale study should be required. Serum magnesium level should be measured in all cases of acute diarrhea, especially in cases of severe diarrhea with moderate to severe dehydration.

In our study we could not do stool levels of $\mathrm{Mg}^{2+}, \mathrm{K}^{+}, \mathrm{Ca}^{2+}$ and urinary $\mathrm{Mg}^{2+}, \mathrm{K}^{+}$levels to document the loss of $\mathrm{Mg}^{2+}$ and $\mathrm{Ca}^{2+}$ in stool $\& \mathrm{Mg}^{2+}$ and $\mathrm{K}^{+}$loss in urine due to non-availability of these investigations at our c

From our study we conclude that:

\section{Conclusion}

1) In our study the incidence of hypomagnesemia was $55 \%$ in cases of acute diarrhea.

2) There was a significant association between grades of dehydration and severity of hypomagnesemia in acute diarrhea.

3) There was no association between symptoms of hypomagnesemia and serum levels of magnesium with $90 \%$ of cases with mild hypomagnesemia being asymptomatic. However $83.3 \%$ of AGE with severe hypomagnesemia had some symptoms mainly neuromuscular.

4) In acute diarrhea, a significant association was found between hypomagnesemia and hypokalemia. The severity of hypokalemia increased with the severity of hypomagnesemia. In severe hypomagnesemia $83.3 \%$ cases had hypokalemia.

5) Hypocalcemia (total calcium) was associated with hypomagnesemia in acute diarrhea.

6) On the basis of our study it can be recommended that serum magnesium levels must be done in cases of acute diarrhea, especially in situations of

a) Moderate to severe dehydration

b) Moderate to severe hypokalemia or other electrolyte disturbance

c) Symptoms related to hypomagnesemia

d) Abnormal ECG.

\section{Bibiliography}

[1]. F. Richard Bringhurst, Bone and mineral metabolism in health and disease, Harrison's principals of internal medicine $18^{\text {th }}$ edition, volume 2, page 3090-91.

[2]. Elin R J, Assessment of magnesium status, Journal of clinical Chemistry 1987; page 33.

[3]. Saris NE, Mervaala E, Karen H, et al Magnesium An update on physiological, clinical and analytical disease aspects. Journal of clinical chemistry A Jan 2000; 294 page 1-26.

[4]. Al-Ghamdi SM, Cameron EC, Sutton RA. Magnesium deficiency: pathophysiologic and clinical overview. Am J Kidney Dis 1994; $24:$ 737-752.

[5]. R Swaminathan. Magnesium metabolism and its disorders. Clin Biochem Rev May 2003; page47-66.

[6]. Elisaf M, Milionis H, Siamopoulos K. Hypomgnesemic hypokalemia and hypocalcemia: clinical and laboratory characteristic. Mineral electrolyte metabolism. Canadian journal of medicine. 1997; 23 page 105-12.

[7]. Altura BM. Basic biochemistry and physiology of magnesium: a brief review. Mag Tr Ele 1991; 10: $167-171$.

[8]. Kroll MH, Elin RJ. Relationships between magnesium and protein concentrations in serum. Clin Chem 1985; $31: 244-246$. 